\title{
Denosumab for the treatment of osteoporosis
}

\author{
Shyh Poh Teo ${ }^{\mathrm{a}^{*}}$ \\ ${ }^{a}$ Geriatrics and Palliative Unit, Department of Internal Medicine, Raja Isteri Pengiran Anak Saleha Hospital, Jalan \\ Putera Al-Muhtadee Billah, Bandar Seri Begawan, BA1710, Brunei Darussalam.
}

\begin{abstract}
Denosumab is a monoclonal antibody that binds to the receptor activator of nuclear factor- $\kappa B$ ligand (RANKL), thereby reducing osteoclastic activity and bone turnover. The Fracture REduction Evaluation of Denosumab in Osteoporosis every 6 Months (FREEDOM) trial showed a significant reduction in the relative risk of fractures with increased bone mineral density (BMD) due to denosumab. A trial of DenosumAb versus placebo in Males with Osteoporosis (ADAMO) demonstrated a similar effect of denosumab for improving BMD and reducing bone turnover in men. However, concerns were identified regarding an increased risk of fractures upon denosumab cessation. Until further evidence is available, bisphosphonate treatment is recommended to attenuate this risk associated with denosumab discontinuation.
\end{abstract}

Keywords: Denosumab, fracture, osteoporosis

Denosumab is a fully human monoclonal antibody used for osteoporosis treatment. It has a high affinity and specificity for the receptor activator of nuclear factor- $\kappa B$ (RANK) ligand, a cytokine and mediator of osteoclastic bone resorption. RANK is a receptor located on osteoclastic precursors, and binding of the RANK ligand (RANKL) promotes differentiation, proliferation, and survival of osteoclasts, resulting in increased bone turnover. The discovery of this pathway has led to the consideration of new molecular targets for osteoporosis treatment. Denosumab inhibits RANKL, resulting in reduced osteoclastic activity and bone resorption [1]. Although it is administered as a subcutaneous injection every six months, the Denosumab Adherence Preference Satisfaction (DAPS) study showed that $92.4 \%$ of patients preferred denosumab over oral alendronate, in agreement with their associated compliance and satisfaction rates [2].

The landmark trial confirming the benefit of denosumab in osteoporosis treatment was the Fracture REduction Evaluation of Denosumab in Osteoporosis every 6 Months (FREEDOM) trial, which included 7808 osteoporotic older women randomized to denosumab or placebo for

\footnotetext{
* Corresponding author: Shyh Poh Teo

Mailing address: Geriatrics and Palliative Unit, Department of Internal Medicine, Raja Isteri Pengiran Anak Saleha Hospital, Jalan Putera Al-Muhtadee Billah, Bandar Seri Begawan, BA1710, Brunei Darussalam.

Email: shyhpoh.teo@moh.gov.bn

Received: 05 July 2020 / Accepted: 18 August 2020
}

36 months. Denosumab was associated with relative risk reductions of new radiographic vertebral fractures $(68 \%)$, non-vertebral fractures (20\%), and hip fractures (40\%) [3]. Extension of the FREEDOM study showed that the benefits of denosumab treatment continued for up to ten years in terms of low fracture incidence and increased BMD without a plateau and with low rates of adverse events [4]. However, subgroup analyses showed that the risk reduction was significant only in women with a baseline femoral neck bone mineral density (BMD) T-score $\leq-2.5$, a body mass index $(\mathrm{BMI})<$ below $25 \mathrm{~kg} / \mathrm{m} 2$, and without a prevalent vertebral fracture [5].

The FREEDOM study also showed that denosumab was associated with reductions in serum Ctelopeptide of type I collagen (CTX), a biochemical marker of bone resorption, to below premenopausal reference intervals [6]. Improvements were also noted in hip and spine strength, by $14.3 \%$ and $22.4 \%$, respectively, at 36 months compared to placebo, as measured through nonlinear finite element analysis (FEA) of hip and spine quantitative computed tomography (QCT) scans [7]. The increased hip and spine strength was seen in both the trabecular and cortical bone components. The benefits of denosumab were also demonstrated in men with low BMD in the A trial of DenosumAb versus placebo in Males with Osteoporosis (ADAMO). When 228 men were randomized to denosumab or placebo for 24 months, the treatment was associated with an increased BMD and reduced bone resorption at levels similar to the FREEDOM study [8].

Two separate studies from Sweden and the United States also showed denosumab to be more cost effective than other osteoporotic agents, including bisphosphonates and 
teriaparatide, for treatment of older men aged over 75 years. The lifetime cohort Markov model demonstrated that denosumab had a higher annual treatment cost compared to other medications but was more cost effective for osteoporosis treatment in terms of the lifetime expected costs and quality-adjusted life-years [9, 10].

A meta-analysis identified ten randomized controlled trials that compared the efficacy of denosumab versus bisphosphonates. Denosumab was associated with a significant improvement in BMD at the lumbar spine, hip and femoral neck at 12 and 24 months compared to bisphosphonates. A lower incidence of osteoporotic fractures was also evident for denosumab than for alendronate at 24 months [11]. Another trial confirmed a significant improvement in spine and hip BMD with denosumab versus risedronate at 24 months, with no difference in adverse events, in patients with glucocorticoid-induced osteoporosis [12].

A case series of severe spontaneous vertebral fractures after denosumab discontinuation raised concerns regarding a severe osteoporosis rebound upon treatment cessation [13]. An observational study found a reversal of BMD benefits and a possibly increased fracture risk, as eight (9.8\%) patients experienced 17 fractures within the year [14]. By contrast, a 24-month follow-up of the FREEDOM study participants who discontinued denosumab revealed similar fracture rates in the denosumab (7\%) and placebo $(9 \%)$ groups, with no evidence of any differences in the fracture occurrence patterns [15]. However, until further evidence is available, the most prudent recommendation is to assess the risks and benefits of denosumab treatment after five years and, if denosumab is to be discontinued, to consider bisphosphonates to attenuate rebound increases in bone turnover [16]. Further studies are required to explore the optimal duration of prolonged denosumab treatment and which anti-resorptive agent is preferable after discontinuation of denosumab.

Finally, given the possible rebound in fracture risk with denosumab cessation, clinicians should be vigilant and emphasize the importance of patient compliance with treatment. A Swedish Prescribed Drug Register identified multiple reasons that may contribute to reduced adherence to treatment, including the healthcare organization, approaches to drug monitoring, and population disease awareness [17]. Thus, a systematic healthcare approach is also required to avoid premature discontinuation of osteoporosis treatment, and particularly denosumab.

In summary, denosumab is useful for osteoporosis management, but clinicians should be aware of the increased fracture risk upon treatment discontinuation.

\section{Declaration}

Conflict of Interest: The authors declare that they have no conflict of interest.

\section{References}

1. Lewiecki EM. RANK ligand inhibition with denosumab for the management of osteoporosis. Expert Opin Biol Ther 2006: 6(10):1041-1050.

2. Freemantle N, Satram-Hoang S, Tang ET, et al. Final results of the DAPS (Denosumab Adherence Preference Satisfaction) study: a 24-month, randomized, crossover comparison with alendronate in postmenopausal women. Osteoporos Int 2012: 23:317-326.

3. Cummings SR, San Martin J, McClung MR, et al. Denosumab for prevention of fractures in postmenopausal women with osteoporosis. N Engl J Med 2009: 361:75665.

4. Bone HG, Wagman RB, Brandi ML, et al. 10 years of denosumab treatment in postmenopausal women with osteoporosis: results from the phase 3 randomised FREEDOM trial and open-label extension. Lancet Diabetes Endocrinol 2017: 5(7):513-523.

5. McClung MR, Boonen S, Torring 0, et al. Effect of denosumab treatment on the risk of fractures in subgroups of women with postmenopausal osteoporosis. J Bone Miner Res 2012: 27(1):211-218.

6. Eastell R, Christiansen C, Grauer A, et al. Effects of denosumab on bone turnover markers in postmenopausal osteoporosis. J Bone Miner Res 2011: 26(3):530-537.

7. Keaveny TM, McClung MR, Genant HK, et al. Femoral and vertebral strength improvements in postmenopausal women with osteoporosis treated with Denosumab. J Bone Miner Res 2014: 29(1):158-165.

8. Langdahl BL, Teglbjaerg CS, Ho PR, et al. A 24-month study evaluating the efficacy and safety of denosumab for the treatment of men with low bone mineral density: results from the ADAMO trial. J Clin Endocrinol Metab 2015: 100(4):1335-1342.

9. Parthan A, Kruse M, Agodoa I, Silverman S, Orwoll E. Denosumab: a cost-effective alternative for men with osteoporosis from a Swedish payer perspective. Bone 2014;59:105-113.

10. Silverman S, Agodoa I, Kruse M, Parthan A, Orwoll E. Denosumab for elderly men with osteoporosis: a costeffectiveness analysis from the US payer perspective. J Osteoporos 2015;2015:627631

11. Lyu H, Jundi B, Xu C, et al. Comparison of denosumab and bisphosphonates in patients with osteoporosis: a meta-analysis of randomised controlled trials. J Clin Endocrinol Metab 2019;104(5):1753-65.

12. Saag KG, Pannacciulli N, Geusens P, et al. Denosumab versus risedronate in glucocorticoid-induced osteoporosis: final results of a twenty-four-month randomized, double-blind, double-dummy trial. Arthritis Rheumatol 2019;71(7):1174-1184.

13. Aubry-Rozier B, Gonzalez-Rodriguez E, Stoll D, Lamy O. Severe spontaneous vertebral fractures after denosumab discontinuation: three case reports. Osteoporos Int 2015: 27:1923-1925.

14. McClung MR, Wagman RB, Miller PD, Wang A, Lewiecki EM. Observations following discontinuation of long-term denosumab therapy. Osteoporos Int 2017: 28:17231732. 
15. Brown JP, Roux C, Torring 0, et al. Discontinuation of denosumab and associated fracture incidence: analysis from the Fracture Reduction Evaluation of Denosumab in Osteoporosis Every 6 Months (FREEDOM) trial. J Bone Miner Res 2013: 28(4):746-752.

16. Tsourdi E, Langdahl B, Cohen-Solal M, et al. Discontinuation of denosumab therapy for osteoporosis: a system- atic review and position statement by ECTS. Bone 2017: 105:11-17.

17. Karlsson L, Lundkvist J, Psachoulia E, Intorca M, Strom 0. Persistence with denosumab and persistence with oral bisphosphonates for the treatment of postmenopausal osteoporosis: a retrospective, observational study, and a meta-analysis. Osteoporos Int 2015;26:2401-2411.

Cite this article as: Teo S P. Denosumab for the treatment of osteoporosis[J]. Aging Pathobiology and Therapeutics, 2020, 2(3): 168-170. 